\title{
Experimental and CFD Investigation on Flow Behaviors of a NPP Pump under Natural Circulation Condition
}

\author{
Weitong Li $(\mathbb{D}$, Lei Yu $\mathbb{D}$, Jianli Hao $\mathbb{D}$, and Mingrui Li $\mathbb{D}$ \\ College of Nuclear Science and Technology, Naval University of Engineering, Wuhan 430033, China \\ Correspondence should be addressed to Lei Yu; yulei301@163.com
}

Received 18 July 2019; Revised 18 September 2019; Accepted 26 September 2019; Published 22 October 2019

Academic Editor: Eugenijus Ušpuras

Copyright (c) 2019 Weitong Li et al. This is an open access article distributed under the Creative Commons Attribution License, which permits unrestricted use, distribution, and reproduction in any medium, provided the original work is properly cited.

\begin{abstract}
Passive safety system is the core feature of advanced nuclear power plant (NPP). It is a research hotspot to fulfill the function of passive safety system by improving the NPP natural circulation capacity. Considering that the flow behaviors of stopped pump pose a significant effect on natural circulation, both experimental and computational fluid dynamics (CFD) methods were performed to investigate the flow behaviors of a NPP centrifugal pump under natural circulation condition with a low flow rate. Since the pump structure may lead to different flows depending on the flow direction, an experimental loop was set up to measure the pressure drop and loss coefficient of the stopped pump for different flow directions. The experimental results show that the pressure drop of reverse direction is significantly greater than that of forward direction in same Reynolds number. In addition, the loss coefficient changes slightly while the Reynolds number is greater than $8 \times 10^{4}$; however, the coefficients show rapid increase with the decrease in Reynolds number under lower Reynolds number condition. According to the experimental data, an empirical correlation of the pump loss coefficient is obtained. A CFD analysis was also performed to simulate the experiment. The simulation provides a good accuracy with the experimental results. Furthermore, the internal flow field distributions are obtained. It is observed that the interface regions of main components in pump contribute to the most pressure losses. Significant differences are also observed in the flow field between forward and reverse condition. It is noted that the local flows vary with different Reynolds numbers. The study shows that the experimental and CFD methods are beneficial to enhance the understanding of pump internal flow behaviors.
\end{abstract}

\section{Introduction}

One significant difference between the proposed advanced reactor and traditional reactor is the design of passive safety system, e.g., passive residual heat removal system (PRHRS). In recent years, great efforts have been made to improve the natural circulation capacity of reactor to fulfill the function of passive safety system. It is noted that key factors restricting the enhancement of natural circulation included heat source power, height difference between cold and heat source, and flow resistance [1]. Accordingly, the researches of thermal-hydraulic behaviors in reactor components are continually implemented to enhance the understanding of natural circulation.

In particular, the flow behaviors of stopped reactor coolant pump (RCP) have gained great attention for a new type of floating nuclear power plant (NFNPP) [2]. NFNPP is a multipurpose plant that offers advantages of simplified loop design, low cost as well as reduced space. It can be applied for offshore oil drilling and island development. The main components in NFNPP consist of reactor, U-tube steam generator (UTSG), RCP, pressurizer, PRHRS, and secondary equipment. The single-phase coolant driving by RCP flows along the primary loop under normal operating conditions, namely forced circulation. However, the RCP stops during a station blackout accident. In that case, the coolant is driven by natural circulation and the stopped RCP converts to a resistance equipment. The flow behaviors of natural circulation are quite different with that of forced circulation, especially for the flow resistance of stopped RCP, which seriously affects the improvement of the natural circulation. In addition, the reverse flow phenomenon in 
primary loop also received substantial interest [3]. The mechanism of reverse flow was explained by many scholars [4], but the solution is not very effective for now. When PRHRS is under operation, the reverse flow rate may be greater than forward flow rate. At this moment, the coolant from the reactor inlet flows through RCP in reverse direction to reactor outlet [5]. The phenomenon leads to the decline of residual heat removal capability.

In fact, it is difficult to obtain the general resistance correlations in theory due to the variation and complexity of the geometry of pump channel. The difficulty leads to the great unpredictability for the reactor safety analysis. According to the present literature, many studies have been reported about various pump behaviors. RCP studies for the integral facilities were summarized by Choi et al. [6] from the standpoint of scaling modeling, single- and two-phase pump behaviors. Wang et al. [7] explored the gas content effect of RCP with power shutting down transitions. Han et al. [8] simulated the coastdown flow of RCP during a loss of heat sink accident. However, the understanding about flow behaviors of RCP under natural circulation condition is insufficient. Especially, the importance of pressure drop and loss coefficient in low Reynolds number for RCP should be fully considered. In spite of the existing deficiency in pump study, a similarity of loss coefficient in low Reynolds number were found in spacer grids, which offer support for rods and temperature measurements in the reactor core. Several scholars explored the flow behaviors for spacer grids in different Reynolds numbers, which are of great significance for reference. Rehme [9] developed a classical pressure drop model for spacer grid to evaluate frictional and form losses respectively. Cigarini and Donne [10] and Schikorr et al. [11] analyzed the applicability of the Rehme model for the various types of spacer grids. Pacio et al. [12] proposed an empirical correlation applicable to the resistance characteristics of 19-pin hexagonal rod. Chenu et al. [13] compared various pressure drop models of spacer grid and applied them to TRACE code. In addition, the CFD method is also utilized to study the flow behaviors. Abbasian et al. [14] and Jin et al. [15] analyzed the influence of CFD turbulence models on flow channel simulation. Batta and Class [16] analyzed the influence of roughness and edge features on the pressure drop of spacer grid.

Proper methods are needed to investigate the flow behaviors of RCP in different Reynolds numbers. As described above, the references of spacer grid provide a solution to the issue. In this paper, experimental and CFD methods were carried out to study the flow behaviors of the NPP centrifugal pump under natural circulation conditions. Problems of loss calculation for pump model were described in detail. An experimental design was conducted to measure the pressure drop in forward/reverse direction and the loss coefficient of the stopped centrifugal pump was obtained. A CFD method based on Reynolds-averaged Navier-Stokes (RANS) models was also utilized to simulate the experiment. A commercial CFD code ANSYS FLUENT was employed to investigate the loss coefficient and explore the internal flow state of centrifugal pump.

\section{Loss Calculation for Pump Model}

The energy loss in the pipe can be divided into two categories, namely friction loss and form loss. The fluid flows through various complex areas in the stopped centrifugal pump, such as the impeller, guide vane. The flow field of pump internal channel is extremely nonuniform under this condition. The total pressure drop can be expressed as

$$
\Delta P=K \frac{\rho w^{2}}{2}=K \frac{(\rho Q)^{2}}{2 \rho A^{2}} .
$$

The loss coefficient in correlation (1) can be rewritten as

$$
K=\frac{2 g A^{2} H}{Q^{2}},
$$

where $K$ is the loss coefficient, $A$ is the flow area of a discharge pipe, $H$ is the head loss, $w$ is the velocity, and $Q$ is the volumetric flow rate.

The accuracy of loss coefficient is of great significance for analyzing the flow behaviors of the whole reactor system. When modelling with best-estimate system code, the pump resistance is usually simulated by establishing a pump characteristic curve, which is different from other components (e.g., pipe, junction). In the model, the pump parameters and the rated values are usually dealt with dimensionless method, namely head ratio $h$, speed ratio $\alpha$, and flow ratio $v$. The dimensionless parameters are converted into the four-quadrant characteristic curve to characterize the pump operating condition, as shown in Figure 1.

The classification of dimensionless head curve is shown in Table 1. On the one hand, the HVN curve represents the forward flow and forward rotation condition, while the HVR curve represents the forward flow and reverse rotation condition. On the other hand, the HVD curve represents the reverse flow and forward rotation condition, while the HVT curve represents the reverse flow and reverse rotation condition. When the pump stops under natural circulation condition, $\alpha / v=0$, which corresponds to the intersection point of HVN and HVR (or HVD and HVT). Above two points represent the forward flow and the reverse flow, respectively. While the dimensionless coordinate $\left(h / v^{2}\right)$ of the corresponding point is expressed as $C$, the relationship between pump dimensionless parameter and loss coefficient can be expressed as

$$
C=\frac{K Q_{\mathrm{R}}^{2}}{2 g A^{2} H_{\mathrm{R}}},
$$

where the subscript $\mathrm{R}$ represents the rated condition.

As can be seen from the above correlations, the pump loss coefficient in the system code is a fixed value. For components with relatively simple shapes, such as sudden expansion/contraction pipes, the loss coefficient can be calculated by theoretical models. However, it is difficult to deduce the loss theoretically due to the complex internal flow structure of pump. In fact, measuring the loss coefficient by experimental method is accurate and effective. In general, only the experiment for the first quadrant curve 


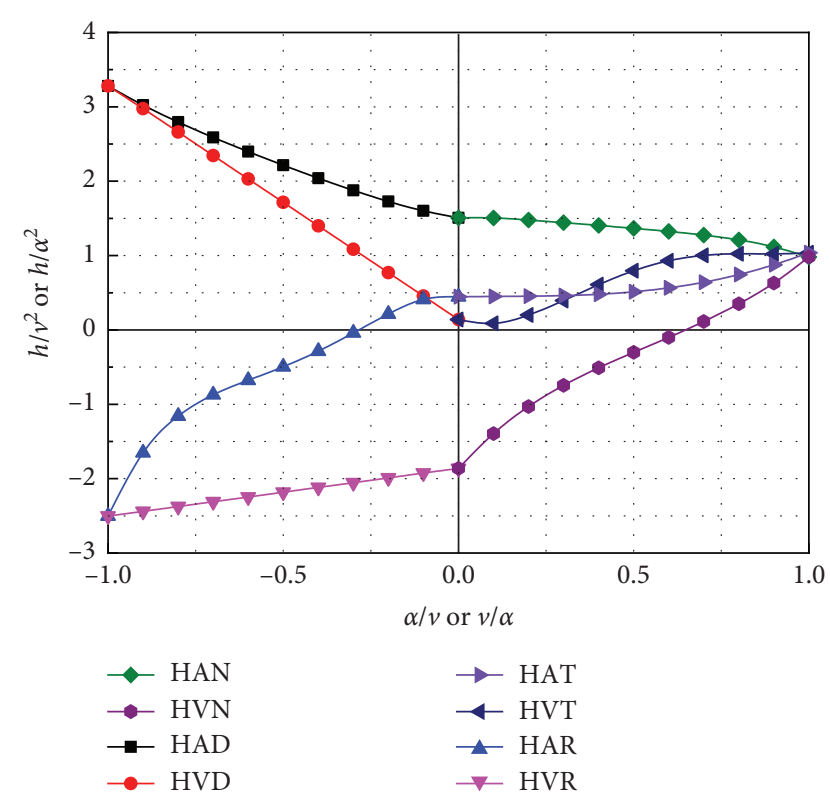

FIGURE 1: Homologous head curve of typical centrifugal pump [17].

Table 1: Classification of centrifugal pump head curve.

\begin{tabular}{lccc}
\hline Pump feature & Head curve & Abscissa & Ordinate \\
\hline \multirow{2}{*}{ Forward flow, forward rotation } & HAN & $v / \alpha$ & $h / \alpha^{2}$ \\
& HVN & $\alpha / v$ & $h / v^{2}$ \\
\multirow{2}{*}{ Reverse flow, forward rotation } & HAD & $v / \alpha$ & $h / \alpha^{2}$ \\
& HVD & $\alpha / v$ & $h / v^{2}$ \\
\multirow{5}{*}{ Reverse flow, reverse rotation } & HAT & $v / \alpha$ & $h / \alpha^{2}$ \\
& HVT & $\alpha / v$ & $h / v^{2}$ \\
\multirow{5}{*}{ Forward flow, reverse rotation } & HAR & $v / \alpha$ & $h / \alpha^{2}$ \\
& HVR & $\alpha / v$ & $h / v^{2}$ \\
\hline
\end{tabular}

(normal operation) of RCPs is completed in practical applications. Sometimes, only the rated data of pump are available; thus, curves from other pumps are used in the calculation. However, the value of $C$ varies greatly for different pumps, which is not conducive for accurate simulation under special conditions (e.g., natural circulation). A few pumps (ATLAS pump [18], etc.) provide the experiment of forward/reverse flow resistance; however, the loss coefficient in low Reynolds number measured in literature [18] was not accurate and no reasonable results were acquired.

\section{Experimental Method and Analysis}

3.1. Experimental Facility. The experimental system consists of the main loop, measurement instrument, water supply/ drainage device, and data acquisition system (DAS). More specifically, the main loop is an open loop, which is mainly composed of auxiliary pump, test centrifugal pump, buffer tank, regulating valve, and corresponding pipeline, as shown in Figure 2. The auxiliary pump in the loop is used to provide a low driving pressure head to simulate the low flow rate condition of natural circulation [19]. The parameters of auxiliary pump are listed as follows:

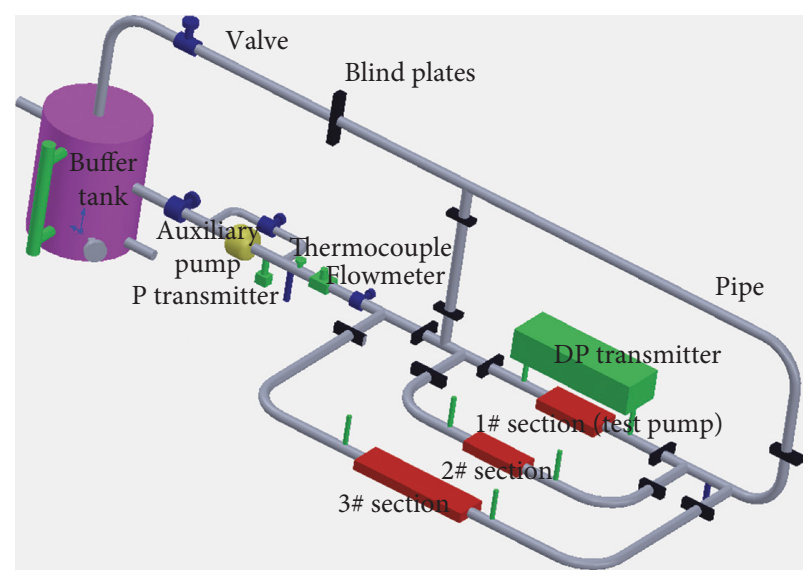

Figure 2: A loop configuration of experimental facility.

$$
\begin{aligned}
Q_{\mathrm{R}} & =20.0 \mathrm{~m}^{3} / \mathrm{h}, \\
N_{\mathrm{R}} & =2900 \mathrm{r} / \mathrm{min}, \\
H_{\mathrm{R}} & =50 \mathrm{~m} .
\end{aligned}
$$

The shaft of test centrifugal pump is fixed. In order to measure the forward and reverse loss coefficient respectively, detachable blind plates are used in the loop to conveniently change the fluid flow direction, as shown in Figure 3. In addition, the test section is divided into multiple branches, so that independent measurements of multiple components can be achieved.

A pressure transmitter is installed at the discharge pipe of the auxiliary pump to monitor the operating condition. A K-type thermocouple is used to measure the fluid temperature of the test section. The flow rate of test section was measured by the electromagnetic flowmeter. The pressure drop of test section is measured by differential pressure (DP) transmitter. Since large collision and disturbance may occur at the pump suction and discharge, the guide pipe of the DP transmitter is set at the position of 10D before the suction and $10 \mathrm{D}$ after the discharge (D: the pipe diameter) in order to reduce the measurement error.

According to the plant's actual natural circulation capability, the Reynolds number is lower than $1.5 \times 10^{5}$. To cover the anticipative operating condition, the experiment condition of Reynolds number range changes from $2.0 \times 10^{4}$ to $1.5 \times 10^{5}$. During the experiment, the pressure drop curve is obtained by controlling the flow rate and temperature, and then the loss coefficient is calculated. In addition, DAS is established based on Labview code and signal conversion device to facilitate the treatment of various measured parameters.

3.2. Uncertainty Analysis. It is impossible to obtain the absolutely true value of the experiment. As a result, the accuracy and dispersion of the experimental data need to be analyzed in the actual situation, namely, the data uncertainty. A root sum square (RSS) method is utilized to evaluate the data uncertainty, which mainly origins from the measurement device, signal conversion device, and DAS [20]. 


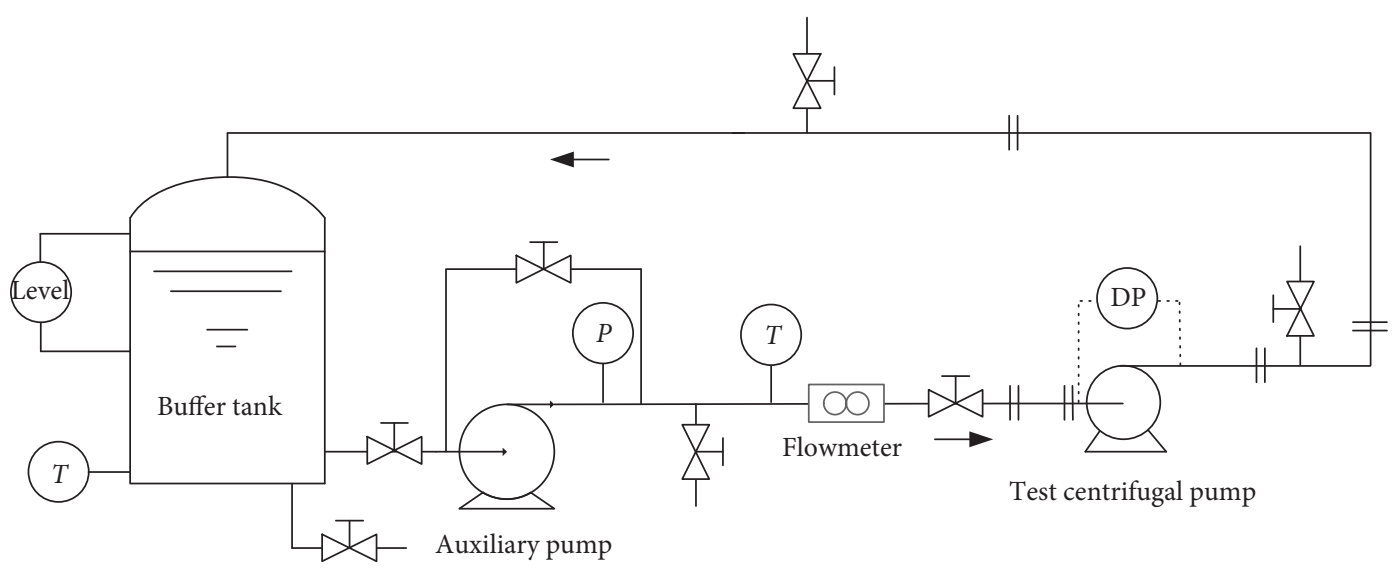

$\mp$ Valve

4 Flow direction

$=$ Blind plates (connected)

(a)

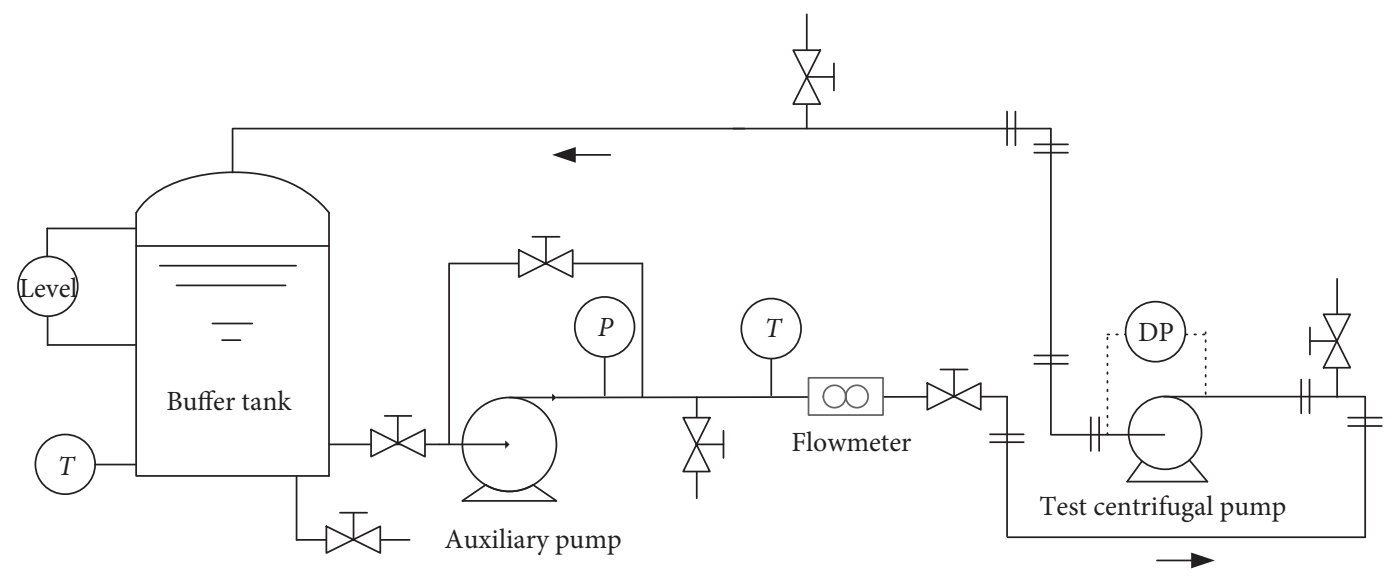

$\searrow$ Valve

4 Flow direction

$=$ Blind plates (connected)

(b)

Figure 3: Measurement diagram in different flow directions. (a) Forward flow. (b) Reverse flow.

The uncertainty of flow rate measurement can be written as follows:

$$
u_{\mathrm{Q}}=\sqrt{u_{\mathrm{f}}^{2}+u_{\mathrm{I} / \mathrm{V}}^{2}+u_{\mathrm{d}}^{2}}=0.6 \%,
$$

where $u_{\mathrm{f}}$ is the uncertainty of flowmeter, $u_{\mathrm{I} / \mathrm{V}}$ is the uncertainty of I/V conversion device, and $u_{\mathrm{d}}$ is the uncertainty of DAS.

The uncertainty of temperature measurement can be written as follows:

$$
u_{T}=\sqrt{u_{\mathrm{th}}^{2}+u_{\mathrm{tt}}^{2}+u_{\mathrm{d}}^{2}}=0.5 \%,
$$

where $u_{\text {th }}$ is the uncertainty of thermocouple and $u_{\mathrm{tt}}$ is the uncertainty of temperature transmitter.

The uncertainty of pressure drop measurement can be written as follows:

$$
u_{P}=\sqrt{u_{\mathrm{pt}}^{2}+u_{\mathrm{I} / \mathrm{V}}^{2}+u_{\mathrm{d}}^{2}}=0.2 \%,
$$

where $u_{\mathrm{pt}}$ is the uncertainty of DP transmitter. 
According to correlation (1), the fluid density error can be ignored due to the change is less than $0.1 \%$. The uncertainty of loss coefficient can be expressed as the indirect uncertainty of pressure drop and flow rate:

$$
u_{K}=\sqrt{\left(\frac{\partial K}{\partial \Delta P}\right)^{2} u_{P}^{2}+\left(\frac{\partial K}{\partial Q}\right)^{2} u_{Q}^{2}}=1.2 \% .
$$

3.3. Experimental Results. The pressure drop characteristics of the stopped centrifugal pump were obtained by controlling the flow rate and temperature. With the increase in flow rate and Reynolds number, the pressure drop curve shows a parabolic growth, and the pressure drop curve obtained for multiple tests is basically consistent, which verifies the repeatability of the experiment.

Figure 4 shows the forward and reverse loss coefficient of the centrifugal pump calculated from the experiment. Reynolds number is calculated according to the reference point in the pump outlet, namely $\operatorname{Re}=\rho v D_{h} / \mu$ [21]. It can be seen that the reverse pressure drop is obviously greater than forward pressure drop under the identical Reynolds number condition. The loss coefficient basically remains unchanged in a high Reynolds number $\left(8 \times 10^{4}\right)$, which is similar with the experimental results in literature [6]. In addition, when the Reynolds number is lower than $8 \times 10^{4}$, the loss coefficient increases significantly with the decline of Reynolds number, up to $20 \%$ at most. However, the measurement of pressure drop for low Reynolds number in literature [6] was inaccurate and the experimental results were not related with correlation clearly.

The empirical correlation of loss coefficient expressed in the form of $a+b / \operatorname{Re}^{c}$ [22] agrees well with the experimental results. The correlation is as follows:

$$
K= \begin{cases}18.5+\frac{2.576 \times 10^{5}}{\operatorname{Re}^{1.094}}, & \text { forward flow, } \\ 25.0+\frac{9.452 \times 10^{3}}{\operatorname{Re}^{0.807}}, & \text { reverse flow, }\end{cases}
$$

where $a$ is the reference constant and $b$ and $c$ are empirical coefficients (Re: $2.0 \times 10^{4} \sim 1.5 \times 10^{5}$ ).

Similar trends are observed in the researches of loss coefficient in spacer grids. Kim et al. [23] obtained a series of empirical correlations based on experiments; however, only the forward flow conditions were investigated. The empirical correlation proposed by Mochizuki is different from the correlation form in this paper, but the range of Reynolds number is similar, and the Reynolds number for transition is also $8 \times 10^{4}$ [24]. The research in literature [20] also shows a rapid decrease in loss coefficient with the increase in Reynolds number under low Reynolds number condition. Therefore, it can be inferred that the pressure drop characteristics of complex structures have certain similarity.

Furthermore, researches indicate that there are a few differences among the loss coefficients of various spacer grids [25]. The correlation of loss coefficient for another NPP pump is also different from correlation (9) based on the experimental facility. However, the correlation form is similar, which illustrates that proper modifications are needed for loss coefficient and correlation (9) is universal. As mentioned in the previous section, the loss coefficient in RELAP5 code is a fixed value, which is not appropriate to the analysis for nature circulation condition. Therefore, the pump model can be modified by referring to the correlation (9).

\section{CFD Method and Analysis}

4.1. CFD Model. The main fluid domain of centrifugal pump, which includes the inlet pipe section, the inlet plenum, the impeller, the guide vane, the outlet plenum and the outlet pipe section, is modelled with SolidWorks code. In order to eliminate the perturbation of inlet and outlet effect on the flow state, the domain in mainstream direction is extended properly from the upstream to downstream of centrifugal pump. Considering the complexity of the research object, hybrid mesh technique is applied by ICEM CFD code to ensure accurate computation and computational effort. On the one hand, a structured mesh is applied for the inlet and outlet pipe sections. On the other hand, an unstructured mesh is applied for the complex pump internal structures. Preliminary analysis indicates that impeller and guide vane areas (as shown in Figure 5) effect the calculation results greatly; thus, meshes in above areas are densely generated. In the modelling process, the grid test is performed to check the effect of the mesh on pressure drop. First, different lengths of structured mesh for extended pipe are compared for mesh independency. The lengths are divided to $1 \mathrm{D}$ (D: the pipe diameter), 10D, 20D and 40D, respectively. The results indicate that the difference of pressure drop between $20 \mathrm{D}$ and $40 \mathrm{D}$ is less than $5 \%$. Therefore, 20D mesh is chosen for extended pipe. Second, the grid cell effect of the pump fluid domain is analyzed in the test. According to the mesh size controlling, the numbers of mesh cells for the test are $1.0 \times 10^{6}$ (case 1), $2.2 \times 10^{6}$ (case 2 ), and $3.1 \times 10^{6}$ (case 3), respectively. The meshes in case 1 are rough, resulting to a larger pressure drop. The prediction between case 2 and case 3 is similar within 3\%. Therefore, the total number of meshes is finally determined as $2.2 \times 10^{6}$ (as shown in Figure 6).

The simulations employ a commercial CFD code ANSYS FLUENT. Literature [20] indicates that turbulence models are of great importance in the simulation. Turbulence models based on RANS correlations are applied in this paper since the Reynolds number of the experiment is greater than $2 \times 10^{4}$. After preliminary calculation, the SST $k-\omega$ model, RNG $k-\varepsilon$ model, and standard $k-\varepsilon$ model are compared to evaluate the CFD method. Mass flow rate inlet and pressure outlet boundary condition and a no-slip wall condition are assumed for the calculation. The calculation is iterated continuously until the dimensionless residuals are less than $10^{-4}$ and the constant value of mass flow rate is obtained.

4.2. CFD Results. Figure 7 shows the comparison between CFD calculation and experiment. The CFD methods of three turbulence models predict a slightly higher loss coefficient 


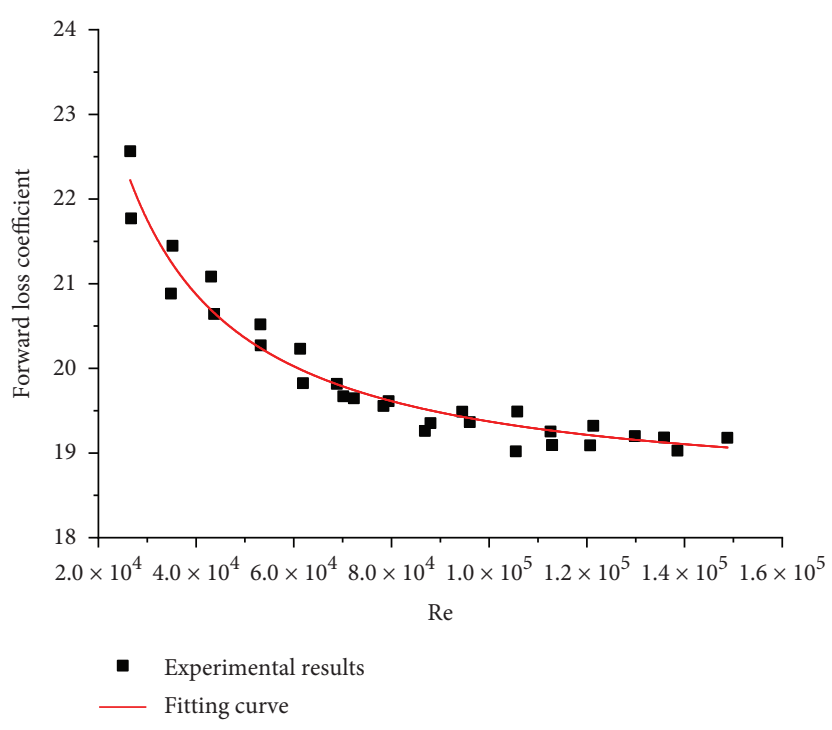

(a)

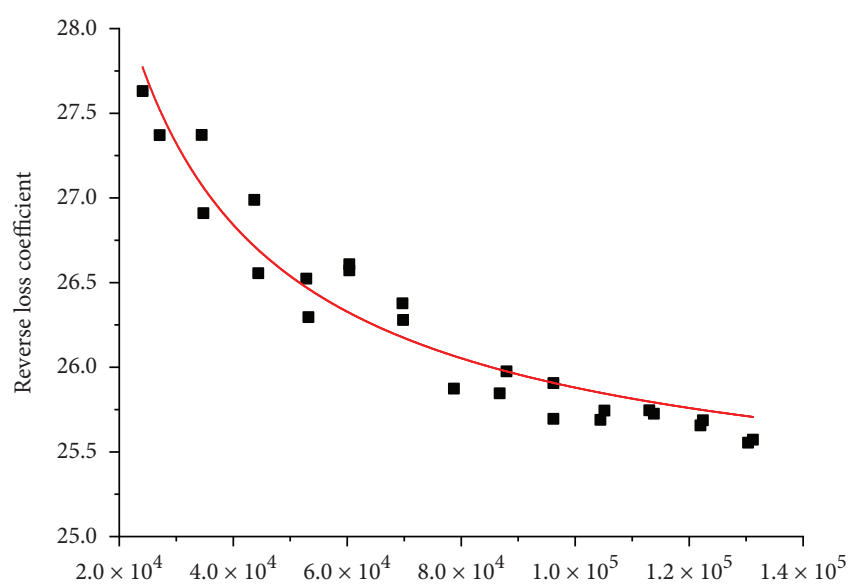

Re

- Experimental results

— Fitting curve

Figure 4: Comparison of loss coefficient in different flow directions. (a) Forward flow. (b) Reverse flow.

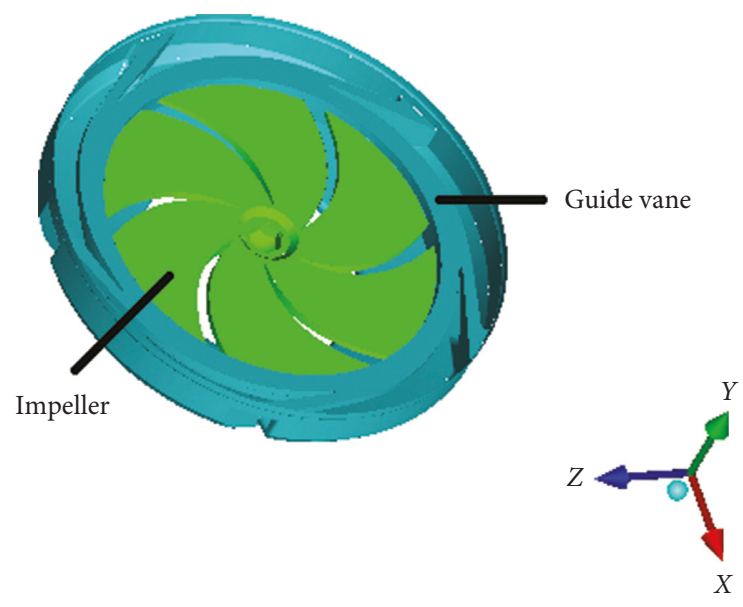

Figure 5: Diagram of impeller and guide vane areas.

than the experimental values and the error of forward calculation is larger than that of reverse calculation. Relatively speaking, the calculation of RNG $k-\varepsilon$ model agrees with the experiment better within $10 \%$ error. Therefore, RNG $k-\varepsilon$ model is performed in the further analysis. As CFD method can reproduce the experimental results and reflect the complex structure feature, it is particularly suitable for predicting flow behaviors in pump optimization design.

Figure 8 shows the streamline and velocity distribution of forward and reverse flows $\left(\operatorname{Re}=1.2 \times 10^{5}\right)$. The values of velocity are normalized with the same scale and the main channels are indicated for convenient comparison. As shown in the figure, the velocity distribution of the reverse flow is more nonuniform. The slamming and backflow of the fluid are prominent, leading to the flow obviously separated from the main flow at the center and the end area of the vane. A relatively high local velocity appears along the edge position. However, the distribution of the forward flow in the

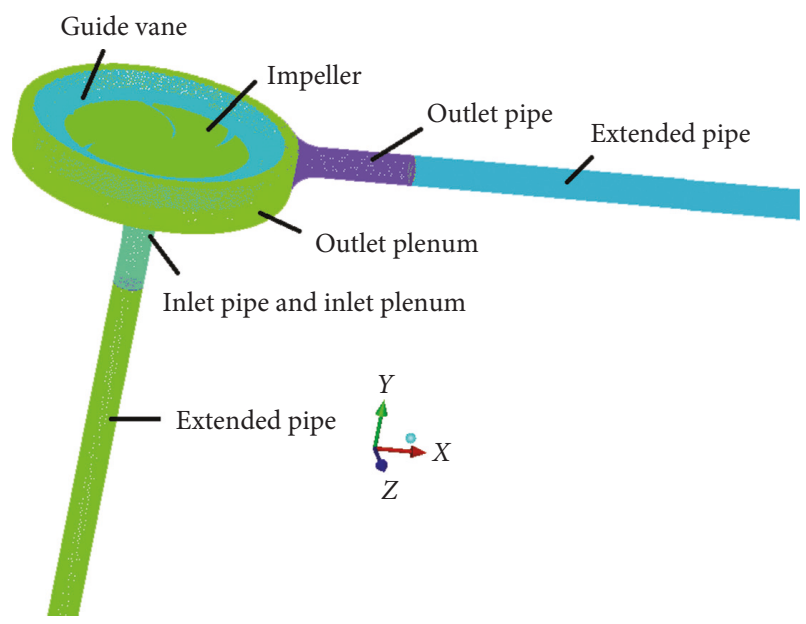

Figure 6: Mesh distribution of main fluid domain.

impeller area is smooth, and velocity changes significantly only in the guide vane area.

Figure 9 shows the pressure distribution of forward and reverse flows $\left(\operatorname{Re}=1.2 \times 10^{5}\right)$. The values of pressure are normalized with the same scale and the main channels are indicated for convenient comparison. Significant pressure drop is observed in the interface area of the main flow channel, e.g., the outlet plenum, guide vane, and impeller. In addition, the pressure distribution of forward and reverse flow is obviously different. The direction of pressure drop in the forward flow is basically opposite to that of reverse flow. According to the color change and scale value described in Figure 9, the forward loss is mainly concentrated in the guide vane channel while the loss in the impeller area accounts for less than $10 \%$. However, reverse loss in the impeller area is more than $20 \%$ for the total loss. Excessively narrow channel of guide vane and change of flow direction lead to the phenomenon. 


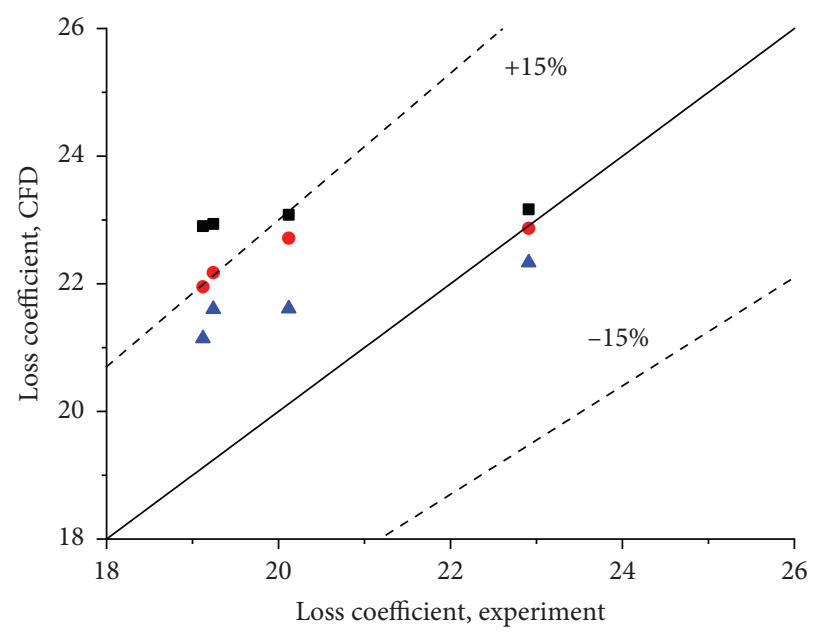

- Forward flow, standard $k$ - $\varepsilon$

- Forward flow, SST $k-\omega$

A Forward flow, RNG $k-\varepsilon$

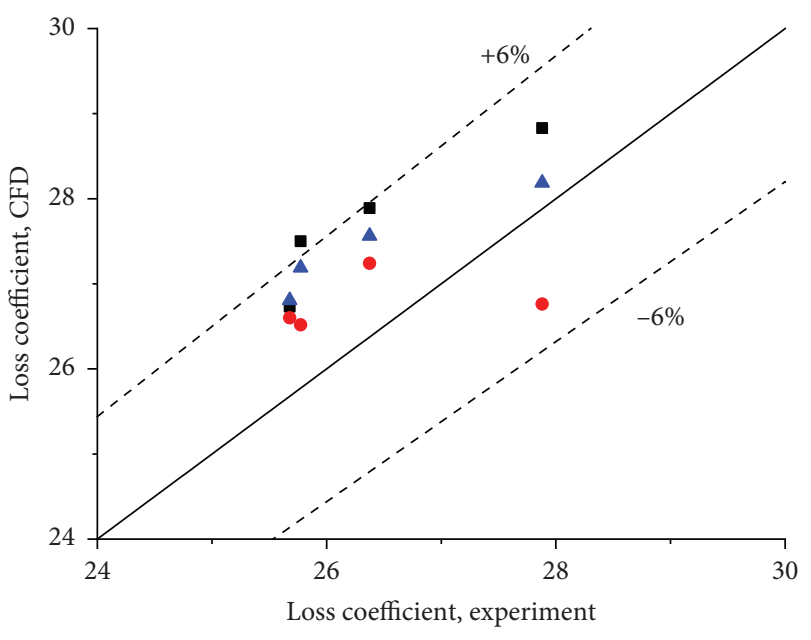

- Reverse flow, standard $k-\varepsilon$

- Reverse flow, SST $k-\omega$

- Reverse flow, RNG $k-\varepsilon$

(a)

(b)

Figure 7: Comparison of CFD calculation and experimental data. (a) Forward flow. (b) Reverse flow.

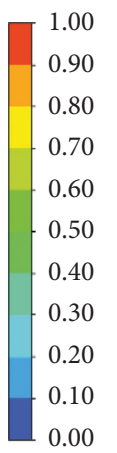

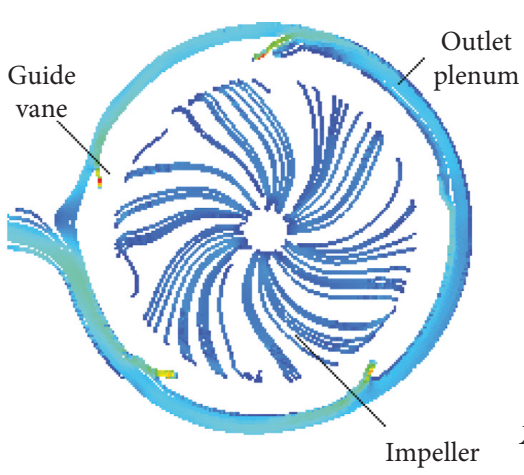

(a)
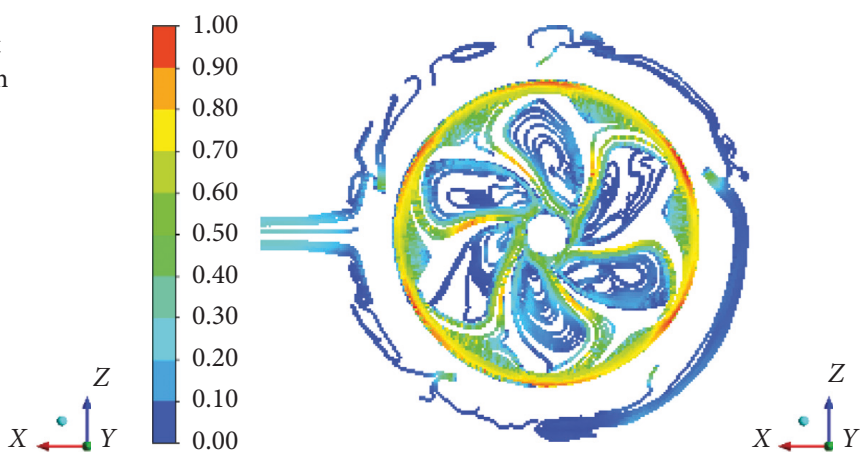

(b)

Figure 8: Comparison of streamline and velocity distribution in different flow directions. (a) Forward flow. (b) Reverse flow.
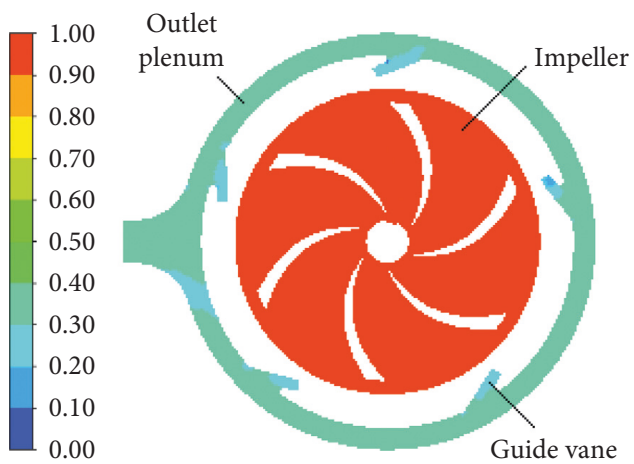

(a)

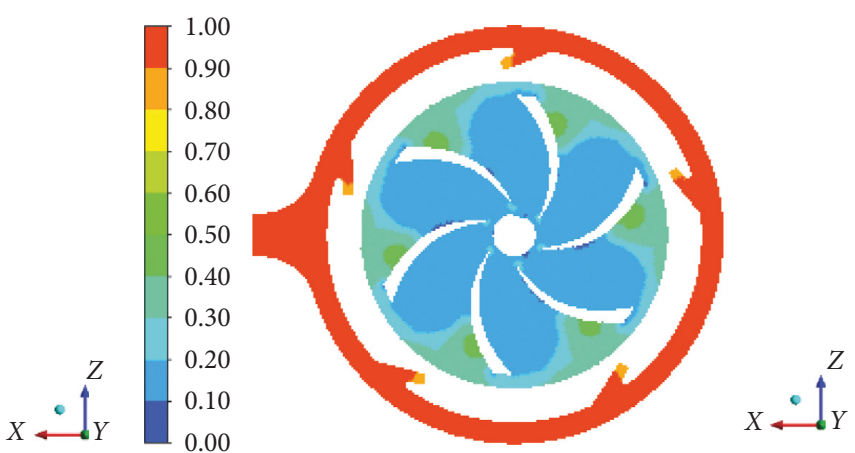

(b)

Figure 9: Comparison of pressure distribution in different flow directions. (a) Forward flow. (b) Reverse flow.

The phenomenon that reverse pressure drop is higher than forward pressure drop occurs in the experiment, and CFD calculation explains that the variation of pump internal flow channel is the root cause. The asymmetry of flow channel, narrow channel of guide vane, and complex variation of impeller lead to the phenomenon of swirl and 

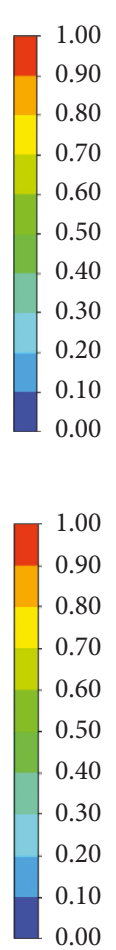

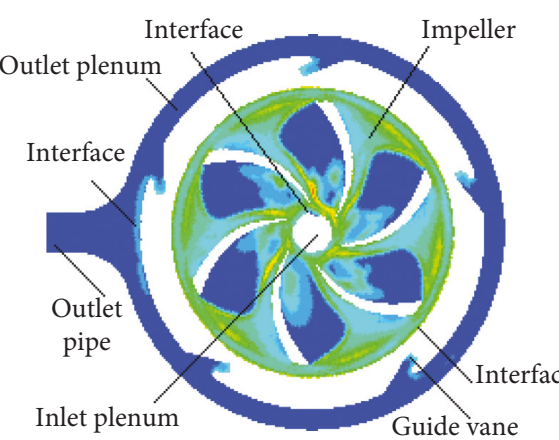

(a)

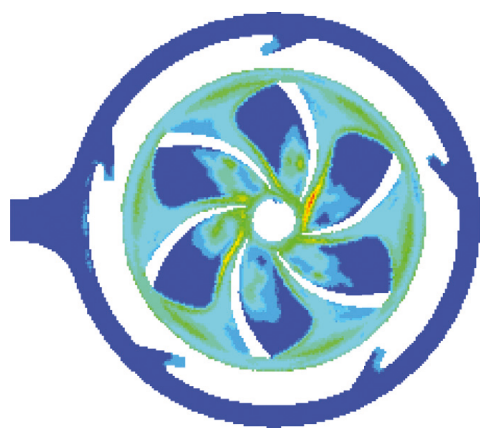

(c)

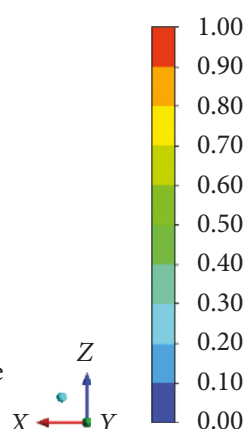

0.00

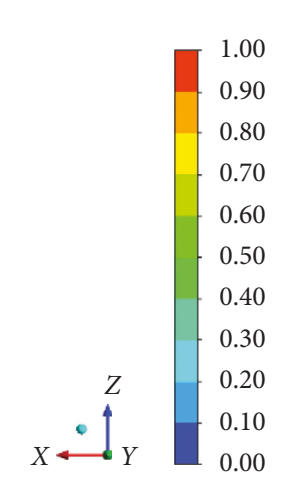

0.00

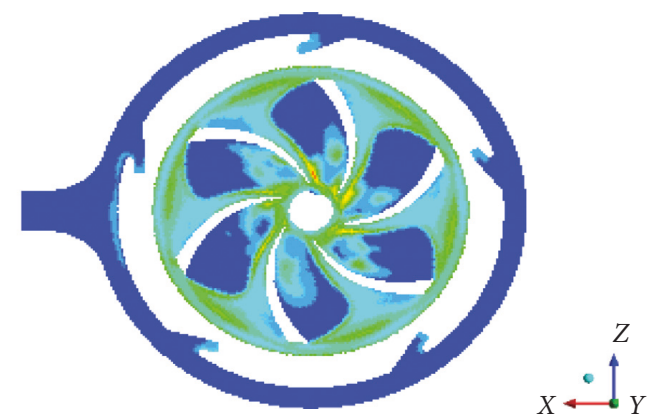

(b)

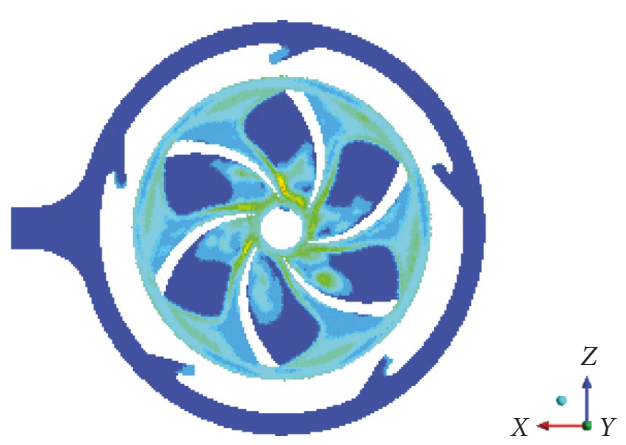

(d)

Figure 10: Comparison of turbulent kinetic energy distribution in different Reynolds numbers. (a) $\operatorname{Re}=2.2 \times 10^{4}$. (b) $\operatorname{Re}=6.0 \times 10^{4}$. (c) $\operatorname{Re}=1.2 \times 10^{5}$. (d) $\operatorname{Re}=1.5 \times 10^{5}$.

backflow. Moreover, the blockage conditions between forward and reverse flow channels are different; thus, the velocity field is greatly different, which changes the apply work of fluid and the pressure loss distribution.

The trend that the loss coefficient increases significantly with the decline of Reynolds number occurs in the experiment, and CFD calculation indicates that local flows differ in various Reynolds number. Taking reverse flow as an example, Figure 10 shows the distribution of turbulent kinetic energy in different Reynolds numbers. The values of turbulent kinetic energy are normalized with the same scale for convenient comparison. It can be seen from Figure 10 that in a low Reynolds number, there is a local high turbulent kinetic energy (the significant change of color and value) in the interface area between outlet plenum and outlet pipe, interface area between guide vane and impeller, indicating that the turbulence intensity of flow in above areas increases greatly and the energy loss is relatively large. The phenomenon of local high turbulent kinetic energy weakens with the increase in Reynolds number, indicating that the complex flow channel of pump has a greater impact on the fluid under low flow rate condition. In this case, the local flow is more unstable in the low Reynolds number, leading to a higher loss coefficient.

\section{Conclusions}

In view of the issues caused by the pump resistance for natural circulation, experimental and numerical methods were developed to investigate the flow behaviors of a NPP centrifugal pump for low flow rate condition. Conclusions can be drawn as follows.

(1) The measured pressure drop of the centrifugal pump indicates a parabolic growth with the increase in Reynolds number. In the identical Reynolds number, the reverse pressure drop is significantly greater than the forward pressure drop. The loss coefficient is estimated to be a constant value while the Reynolds number is larger than $8 \times 10^{4}$.

(2) The loss coefficient tends to increase with the decrease in Reynolds number in low Reynolds number. The phenomenon is similar to the trend of loss coefficient in spacer grids. Based on the experimental results, the empirical correlation of loss coefficient for centrifugal pump is obtained.

(3) The CFD methods predict the loss coefficient well compared with the experiment. The calculation of RNG $k-\varepsilon$ model agrees with the experimental value within $10 \%$ error.

(4) The internal flow field distributions are obtained using CFD methods. The pressure drop of centrifugal pump is mainly concentrated in the interface area of impeller and guide vane, etc. Significant difference is also observed in the flow field between forward and reverse condition, which explains the different pressure losses in the experiment. In addition, local flows are more unstable in low Reynolds numbers. 
The research results in this paper can enhance the understanding of flow behaviors of centrifugal pump under natural circulation condition. It is beneficial to the optimization design of centrifugal pump and the improvement of calculation model. More accurate analysis can be carried out to investigate the feature factors based on specific pump geometry.

\section{Nomenclature}

ATLAS: Advanced thermal-hydraulic test loop for accident CFD: Computational fluid dynamics

DAS: Data acquisition system

DP: Differential pressure

NPP: Nuclear power plant

PRHRS: Passive residual heat removal system

RANS: Reynolds-averaged Navier-Stokes

RCP: Reactor coolant pump

Re: $\quad$ Reynolds number

RSS: $\quad$ Root sum square

UTSG: U-tube steam generator.

\section{Data Availability}

The data used to support the findings of the study are included within the article. Researchers can verify the results and replicate the analysis.

\section{Conflicts of Interest}

The authors declare that there are no conflicts of interest regarding the publication of this paper.

\section{Acknowledgments}

This research was supported by the National Natural Science Foundation of China (No. 11502298).

\section{References}

[1] TECDOC-1281, Natural Circulation Data and Methods for Advanced Water Cooled Nuclear Power Plant Designs, International Atomic Energy Agency, Vienna, Austria, 2002.

[2] Y. Rao, L. Yu, W. Li, and F. Zhang, "A choice of pure steam vertical in-tube condensation model for simulating a passive residual heat removal system," Nuclear Engineering and Design, vol. 293, no. 1, pp. 112-118, 2015.

[3] J. Hao, W. Chen, and D. Zhang, "Effect of U-tube length on reverse flow in UTSG primary side under natural circulation," Annals of Nuclear Energy, vol. 56, no. 1, pp. 66-70, 2013.

[4] G. Hu, L. Yu, W. Chen, J. Hao, and X. Chu, "Experimental and numerical investigations on the reverse flow phenomena in UTSGs," Progress in Nuclear Energy, vol. 92, no. 1, pp. 147154,2016

[5] Y. X. Rao, Study on numerical simulation and operation characteristics of passive residual heat removal system under weak driving force conditions, Ph.D. thesis, Naval University of Engineering, Wuhan, China, 2017.

[6] K. Y. Choi, Y. S. Kim, S. J. Yi, and W.-P. Baek, "Development of a pump performance model for an integral effect test facility," Nuclear Engineering and Design, vol. 238, no. 10, pp. 2614-2623, 2008.
[7] X. L. Wang, P. Wang, S. Q. Yuan et al., "Study on flow characteristics of gas-liquid two phase for reactor coolant pump with power shutting down transition process," Atomic Energy Science and Technology, vol. 48, no. 11, pp. 2004-2011, 2014, in Chinese.

[8] J. W. Han, T. H. Lee, J. H. Eoh, and S.-O. Kim, "Investigation into the effects of a coastdown flow on the characteristics of early stage cooling of the reactor pool in KALIMER-600," Annals of Nuclear Energy, vol. 36, no. 9, pp. 1325-1332, 2009.

[9] K. Rehme, "Pressure drop correlations for fuel element spacers," Nuclear Technology, vol. 17, no. 1, pp. 15-23, 1973.

[10] M. Cigarini and M. D. Donne, "Thermohydraulic optimization of homogeneous and heterogeneous advanced pressurized water reactors," Nuclear Technology, vol. 80, no. 1, pp. 107-132, 1988.

[11] M. Schikorr, E. Bubelis, L. Mansani, and K. Litfin, "Proposal for pressure drop prediction for a fuel bundle with grid spacers using Rehme pressure drop correlations," Nuclear Engineering and Design, vol. 240, no. 7, pp. 1830-1842, 2010.

[12] J. Pacio, M. Daubner, F. Fellmoser et al., "Heavy-liquid metal heat transfer experiment in a 19-rod bundle with grid spacers," Nuclear Engineering and Design, vol. 273, no. 1, pp. 33-46, 2014.

[13] A. Chenu, K. Mikityuk, and R. Chawla, "Pressure drop modeling and comparisons with experiments for single-and two-phase sodium flow," Nuclear Engineering and Design, vol. 241, no. 9, pp. 3898-3909, 2011.

[14] F. Abbasian, S. D. Yu, and J. Cao, "Experimental and numerical investigations of three-dimensional turbulent flow of water surrounding a CANDU simulation fuel bundle structure inside a channel," Nuclear Engineering and Design, vol. 239, no. 11, pp. 2224-2235, 2009.

[15] H. Jin, Y. Lee, and N. Park, "CFD analysis on a three by three nuclear fuel bundle with five spacer grid assemblies," Journal of Mechanical Science and Technology, vol. 26, no. 12, pp. 3965-3972, 2012.

[16] A. Batta and A. G. Class, "CFD analysis of pressure drop across grid spacers in rod bundles compared to correlations and heavy liquid metal experimental data," Nuclear Engineering and Design, vol. 312, no. 1, pp. 121-127, 2017.

[17] W. T. Li, L. Yu, and Y. Q. Chen, "Modeling research and simulation of main coolant pump for marine reactor," Ship Science and Technology, vol. 38, no. 4, pp. 77-80, 2016, in Chinese.

[18] K.-Y. Choi, H.-S. Park, S. Cho et al., "Experimental simulation of a direct vessel injection line break of the APR1400 with the ATLAS," Nuclear Engineering and Technology, vol. 41, no. 5, pp. 655-676, 2009.

[19] J. Hao, W. Chen, G. Hu, X. Chu, D. Zhang, and L. Yu, "Experimental research on reverse flow critical point among parallel U-tubes in SG," Progress in Nuclear Energy, vol. 98, no. 1, pp. 59-70, 2017.

[20] W. K. In, C. H. Shin, Y. K. Kwack, and C. Y. Lee, "Measurement and CFD calculation of spacer loss coefficient for a tight-lattice fuel bundle," Nuclear Engineering and Design, vol. 284, no. 1, pp. 153-161, 2015.

[21] T.-H. Chun and D.-S. Oh, "A pressure drop model for spacer grids with and without flow mixing vanes," Journal of Nuclear Science and Technology, vol. 35, no. 7, pp. 508-510, 1998.

[22] A. Epiney, K. Mikityuk, and R. Chawla, "TRACE qualification via analysis of the EIR gas-loop experiments with smooth rods," Annals of Nuclear Energy, vol. 37, no. 6, pp. 875-887, 2010. 
[23] N.-H. Kim, S. K. Lee, and K. S. Moon, "Elementary model to predict the pressure loss across a spacer grid without a mixing vane," Nuclear Technology, vol. 98, no. 3, pp. 349-353, 1992.

[24] TECDOC-1203, Thermohydraulic Relationships for Advanced Water Cooled Reactors, International Atomic Energy Agency, Vienna, Austria, 2001.

[25] S. Cevolani, "ENEA Thermohydraulic Data Base for the Advanced Water Cooled Reactor Analysis," in Proceedings of the 1st Research Co-ordination Meeting of IAEA CRP on Thermohydraulic Relationships for Advanced Water Cooled Reactors, Vienna, Austria, 1995. 

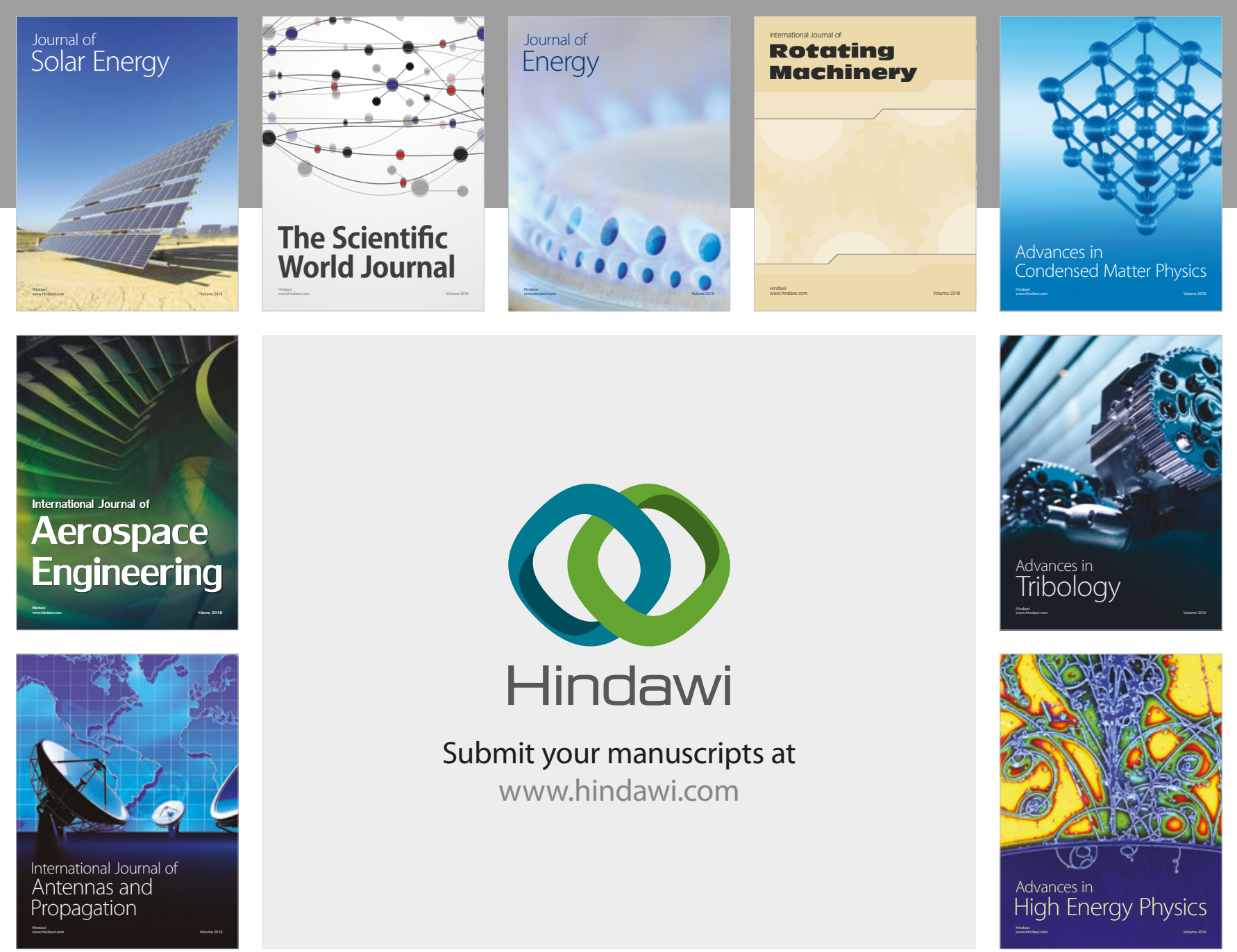

Submit your manuscripts at

www.hindawi.com
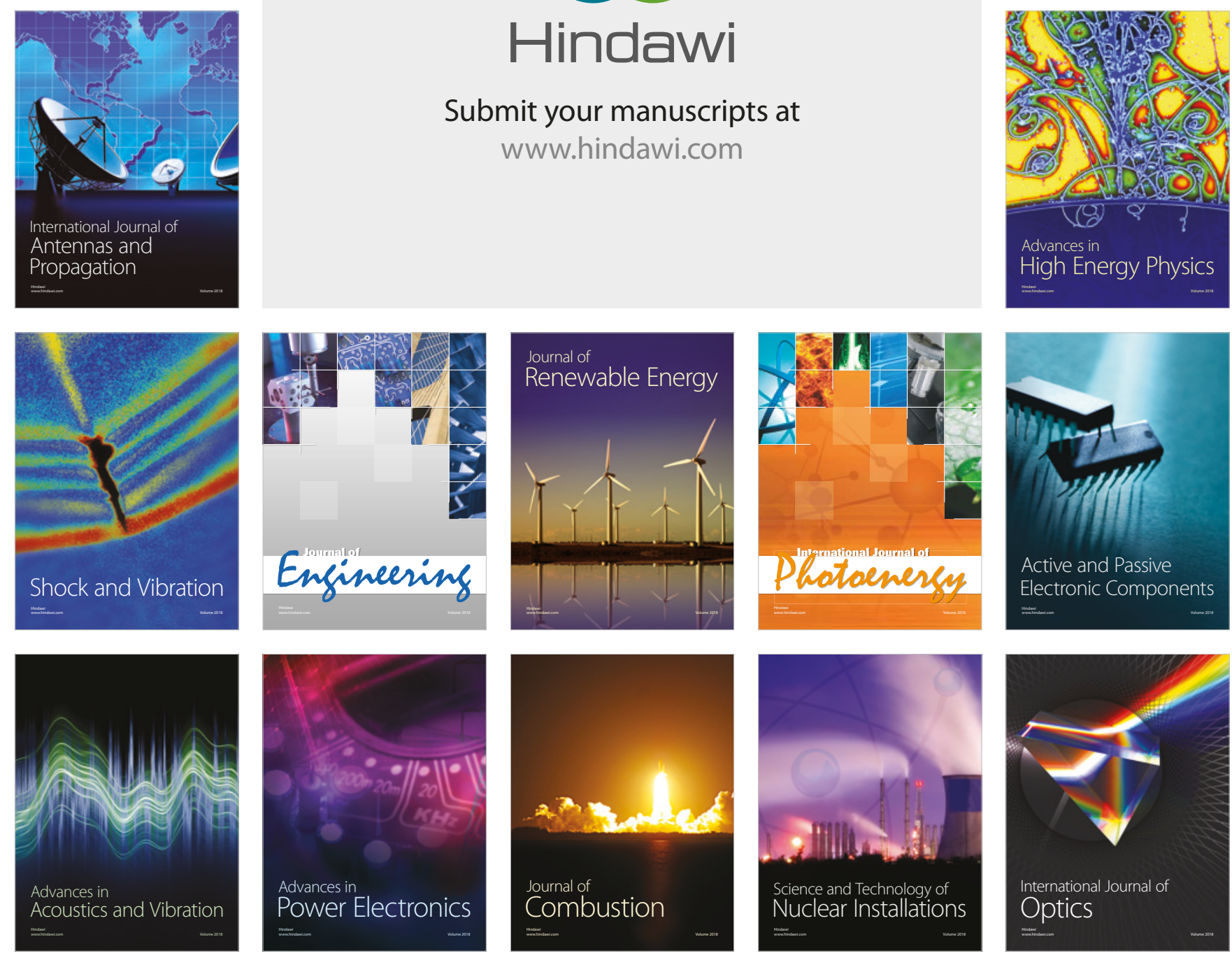